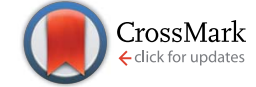

Cite this: RSC Adv., 2017, 7, 9495

Received 4th November 2016

Accepted 28th December 2016

DOI: $10.1039 / c 6 r a 26286 \mathrm{~d}$

www.rsc.org/advances

\section{Facile polypyrrole thin film coating on polypropylene membrane for efficient solar-driven interfacial water evaporation $\dagger$}

\author{
Xiayun Huang, ${ }^{a}$ Yi-Hsin Yu, ${ }^{b}$ Oscar L. de Llergo, ${ }^{a}$ Samantha M. Marquez ${ }^{c}$ \\ and Zhengdong Cheng ${ }^{\star a b d}$
}

\begin{abstract}
We report the fabrication of a polypyrrole-coated interfacial solar membrane using a versatile dip-coating method, which enables solar vapor generation efficiency of $72 \%$ for the purpose of desalination. This nextgeneration method of thermal desalination membrane uses solar thermal energy as a heat source to vaporize salt and brackish water. The polypyrrole thin film is tightly attached to individual nonconductive polypropylene fibers in a mesh. The dip-coating method can be applied to different types of membranes, regardless of the wetting property, conductivity, or surface curvature.
\end{abstract}

Rapid population growth and its attendant demand, climate change, and environmental pollution drives the increasingly serious global challenge of freshwater scarcity. Membrane desalination can play a role in treating non-potable water, including seawater and brackish groundwater, into potable water. ${ }^{1,2}$ Reverse osmosis technology, in which salt or brackish water is forced under pressure through a semipermeable membrane to produce fresh water and a brine waste product, ${ }^{3}$ has emerged as a dominant membrane process for water desalination. ${ }^{1}$ High electrical energy input is needed for power pumps to maintain pressure of $>55$ bar to overcome the osmotic pressure of seawater ${ }^{1,2}$ in a reverse osmosis operation. Our study explores novel technologies to lower the overall energy by employing solar thermal energy for the water desalination by evaporation.

Solar thermal energy, as applied to water desalination, produces fresh water without the large conventional energy demand of large pumps and their consequent carbon emissions. ${ }^{4}$ In nature, water evaporates in the presence of a heat source, such as solar radiation, and condenses in response to a temperature gradient. Early-stage thermal desalination utilized this low-efficiency concept: seawater was heated by electricity and waste heat to cause evaporation, and then condensed at a lower temperature to produce fresh water. ${ }^{4}$

\footnotetext{
${ }^{a}$ Artie McFerrin Department of Chemical Engineering, Texas A\&M University, College Station, TX 77843-3122, USA. E-mail: zcheng@tamu.edu

${ }^{b}$ Department of Materials Science and Engineering, Texas A\&M University, College Station, TX 77843-3003, USA

'Branford College, Yale University, New Haven, Connecticut, 06511, USA

${ }^{d}$ Mary Kay O'Connor Process Safety Center, Artie McFerrin Department of Chemical Engineering, Texas A\&M University, College Station, TX 77843-3122, USA

$\dagger$ Electronic supplementary information (ESI) available. See DOI: $10.1039 / \mathrm{c} 6 \mathrm{ra} 26286 \mathrm{~d}$
}

However, this method consumes a substantial amount of electrical energy. Halas et al. ${ }^{5-7}$ studied a light-induced water purification process. Sunlight irradiates a gold nanoparticle in an aqueous suspension to produce localized heat, and then water vapor evaporates from the individual particles. In this case, heat transfer from the traveling steam bubbles in the bulk water limits evaporation efficiency. ${ }^{8}$ To more efficiently convert the solar energy into heat and to achieve a high water vapor evaporation rate, an interfacial solar membrane was designed to enhance the localized temperature at the water-air interface. This temperature modulation allows more efficient conversion of solar energy into thermal energy by increasing the water evaporation rate at the water-air interface. ${ }^{8}$ The desired membrane is a hydrophobic porous membrane, a property allowing it to float at the water-air interface. At the same time, it has a solar energy absorber layer on the thermal insulation matrix. ${ }^{9-11}$

In this study, we report a versatile thin film dip-coating fabrication strategy for a polypyrrole (PPy)-coated interfacial solar membrane. This material is based on a traditional thermal desalination membrane material, the porous polypropylene membrane, which has been used as a thermal desalination membrane for decades. ${ }^{12}$ Polypyrrole was selected as a thin film coating on the non-conductive polypropylene membrane due to its broad spectrum absorption (from visible to near-infrared) and its high photothermal conversion efficiency. ${ }^{9,13}$ Moreover, PPy has proven to be stable and inexpensive in bioelectronics and biomedical applications compared with noble metal nanoparticles. ${ }^{14}$ It exhibits good biocompatibility and low longterm cytotoxicity ${ }^{13}$ compared with carbon material. ${ }^{15}$ Furthermore, it is also reported that the coating's hydrophobic fluoroalkylsilane top layer imbues the PPy coating with a wettability self-healing property to maintain hydrophobicity. ${ }^{9}$ This self- 
healing property is responsible for the self-migration of fluoroalkylsilane within the PPy coating under chemical oxidation (oxidation occurs under water and air and strong radiation from UV light). Solar vapor generation efficiency is maintained by the membrane floating at the water-air interface. Herein, we prepared the fluoroalkylsilane surface layer of the PPy coating, which is expected to maintain a long-term hydrophobicity. Instead of the electroplated PPy coating technology developed by Wang et al., ${ }^{9}$ which can coat only a conductive substrate (i.e., thermal conductive stainless steel mesh used by Wang et al.), this thin film dip-coating method enables a facile solutionbased fabrication of PPy coating on a variety of substrates, including conductive and non-conductive and curved structures. Herein, we selected thermal insulation polypropylene (PP) mesh, which has historically been used as a thermal desalination membrane. ${ }^{16}$ Our results show that $\sim 300 \mathrm{~nm}$ thick PPy thin film coating on the thermal insulation polypropylene mesh has a solar vapor generation efficiency of $72 \%$, which is substantially higher than that of the commercialized solar stills with typical efficiencies from $24 \%$ to $45 \%$ (ref. 17) and still has slightly higher efficiency than the thicker PPy coating on the thermal conductive stainless steel mesh. ${ }^{9}$

Using our facile dipping strategy, PPy thin film coating is directly deposited by immersing the substrate in a mixture of dilute aqueous solution of pyrrole as a monomer and $\mathrm{FeCl}_{3}$ as an oxidant (Fig. 1A). This solution initiates direct polymerization on the surface and tightly bonds to the substrate surface to form a smooth coating, regardless of initial wetting property, electrical conductivity, and curvature of the substrates. ${ }^{18}$ PPy coatings have been reported to have a good adhesion on a variety of substrates, such as hydrophilic glass (Fig. 1B) and
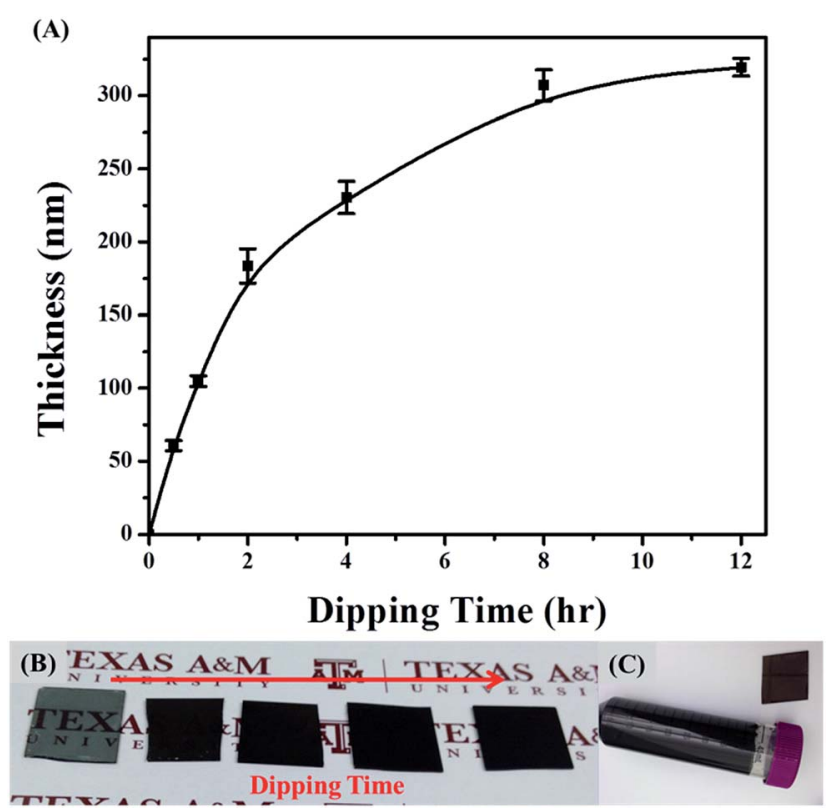

Fig. 1 The growth of PPy coating. (A) Growth of PPy coating on glass substrate with increased dipping time from $0.5 \mathrm{~h}$ to $12 \mathrm{~h}$ (B) PPy coating on the glass with the increasing dipping time (left to right: 0.5 h, 1 h, 4 h, 8 h, and 12 h). (C) PPy coating on glass and polypropylene plastic tube after $12 \mathrm{~h}$. hydrophobic polypropylene plastic centrifuge tubes (Fig. 1C); metal, inorganic and polymer surfaces; as well as on substrates of various curvatures, such as tubing or fibrous structures. ${ }^{19,20}$ In comparison, electrochemical polymerization of PPy, which has been used to form interfacial solar membranes, requires the coating substrate to be able to conduct electricity., ${ }^{\mathbf{9}, 19}$ The electrically conductive substrate has, at the same time, high thermal conductivity as well, which limits the efficiency and spreads out the localized heat. With the increasing reaction time, the thickness of PPy coating increases from $\sim 50 \mathrm{~nm}$ to $\sim 300 \mathrm{~nm}$ (Fig. 1A). Correspondingly, the dark color intensifies with the extended dipping time (Fig. 1B from left to right). Two regions are observed..$^{21}$ The first region is an initial linear growth region that was due to the slow incubation for the deposition in solutions with low oxidizing agent $\left(\mathrm{FeCl}_{3}\right) /$ pyrrole ratios. In the second region, the rate of film growth slows down. This decrease in the deposition rate can be explained by the depletion of the reactants in the solution.

Compared with electrochemical polymerization of polypyrrole, our oxidation polymerization approach is simply based on a facile dipping method that allows for thickness control to the nanometer scale, as well as affording ease of coating on a non-conductive curved surface, such as PP mesh (Fig. 2A) as well as on a conductive curved surface, such as stainless steel mesh (see ESI $\dagger$ ). Scanning electron microscopy images (Fig. 2B) show PPy uniformly coated on each PP fiber in the mesh. At high magnification, the area indicated by the red rectangle revealed that uniform PPy coatings are porous and composed of compacted PPy nanoparticles $(\sim 50 \mathrm{~nm})$. This thin PPy coating (Fig. 2C) exhibits a broad absorption band with a wide range of light from UV to visible light and near infrared, which matches the spectrum of sunlight (orange shadowed). Absorbance increases with the increase in the thickness of the PPy coating. Absorbance of the coating saturates until reaching the $\sim 300 \mathrm{~nm}$ ( 8 h) PPy deposition. Further increasing the thickness of PPy by extending the dipping time (from $8 \mathrm{~h}$ to $12 \mathrm{~h}$ ) does allow harvesting of more solar energy trapped in the PPy membrane.

Surface wetting, another important parameter, influences interfacial solar membrane performance. A hydrophobic surface with excellent water droplet roll-off is the desired property, which depends not only on the chemical nature but also on the hierarchical structure of the surface. ${ }^{22-24}$ Herein, the deposited PPy coating is hydrophilic with a contact angle of nearly $60^{\circ}$ with the flat surface (Fig. 2D). ${ }^{\mathbf{1 9 , 2 0 , 2 5 , 2 6}}$ The hydrophobicity of the PPy coating persists with the increase in the coating thickness from around $50 \mathrm{~nm}$ to $200 \mathrm{~nm}$. To tune the wettability from hydrophilic to hydrophobic, silanization of fluoroalkylsilane by chemical vapor was chosen to uniformly penetrate through the coating, including both the surface layer and the polymer and to voids inside., ${ }^{\mathbf{9} 22}$ After chemical vapor silanization, the PPy coating becomes hydrophobic on the flat surface (Fig. 2E) with good hydrophobicity, as well as enables excellent water droplet roll-off on PP mesh. This desired change in the property is due to the introduction of microscale and nanoscale hierarchical roughness. ${ }^{22}$ A water droplet rolls off from the modified mesh surface easily within $0.1 \mathrm{~s}$, whereas a water droplet of the same volume remains on the hydrophobic 

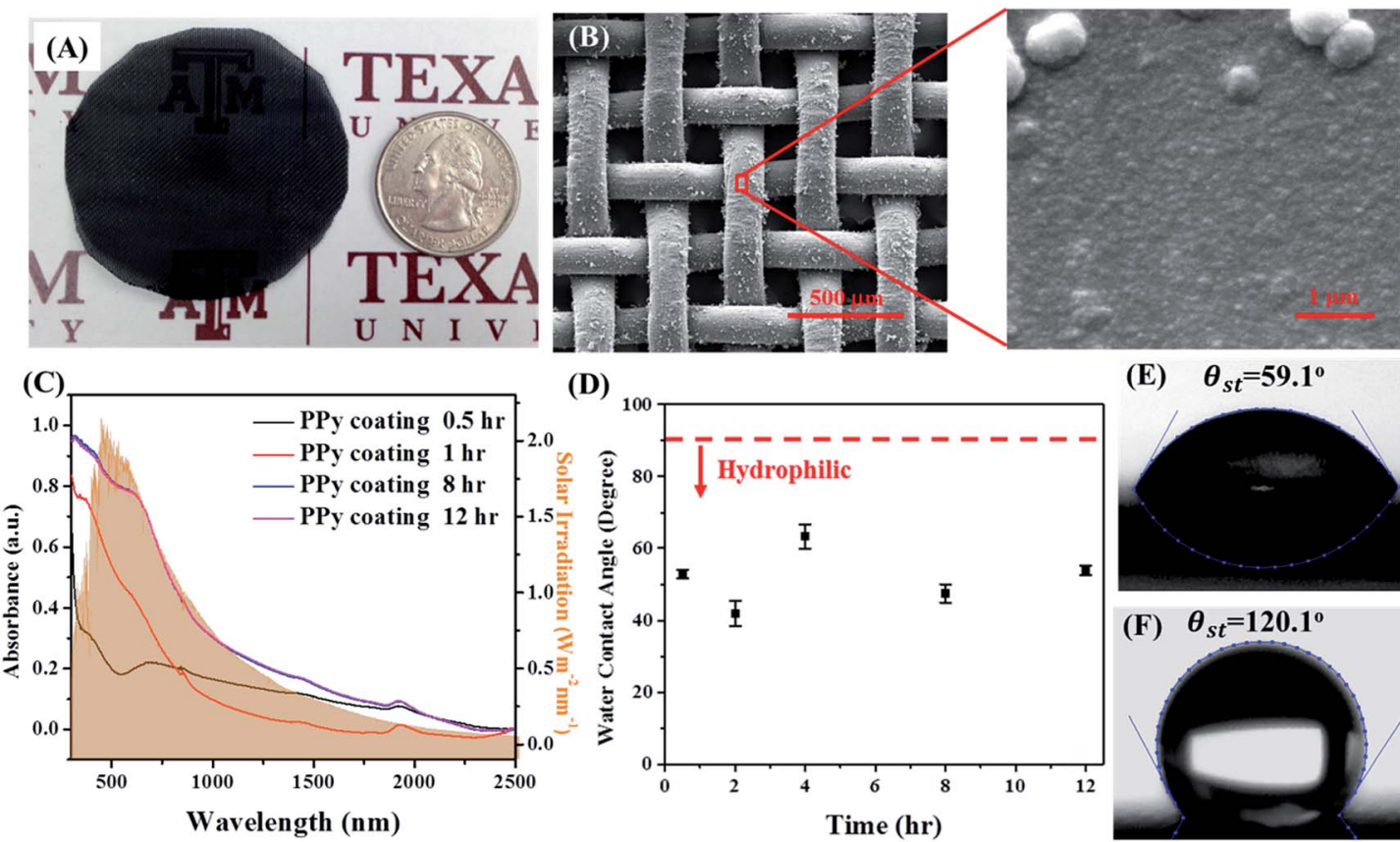

(D)

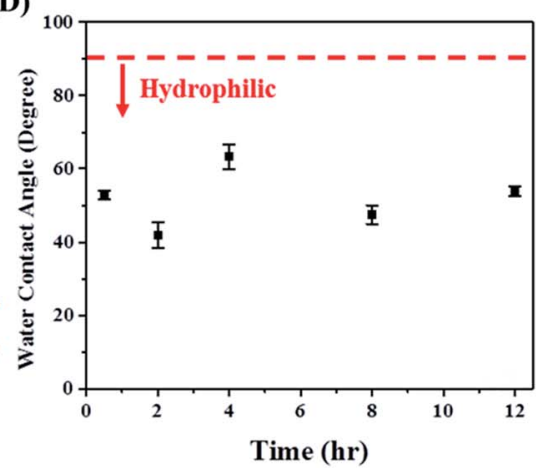

(E) $\boldsymbol{\theta}_{s t}=\mathbf{5 9 . 1 ^ { \circ }}$

Fig. 2 The morphology, water wetting behaviour and solar absorption of PPy coating. (A) Optical image of PPy-coated PP mesh ( 300 nm in thickness) with an area of $861 \mathrm{~mm}^{2}$. (B) SEM images of PPy coating (dipping time, $12 \mathrm{~h}$ ) on the polypropylene mesh (76 mesh with $24 \%$ open pore area and $161 \mu \mathrm{m}$ fiber diameter). The low magnification image (left) shows the PPy coating uniformly coated on each PP fiber. At high magnification (right), the coating is made of the stacked PPy nanoparticles. (C) The absorption of the PPy coating matches the solar spectral irradiance. (D) Water contact angle of hydrophilic PPy coating on glass with increased dipping time from $0.5 \mathrm{~h}$ to $12 \mathrm{~h}$. (E) Static contact angles of a $5 \mu \mathrm{L}$ water drop on the PPy-coated flat surface before and after (F) chemical vapor silanization of fluoroalkylsilane.

PP mesh. (A demonstration video can be found in the ESI. $\dagger$ ) The modified PP mesh has much better water droplet roll-off property, enabling the modified mesh to float on the water surface, compared with using PP mesh alone. Likewise, it can be deposited on various substrates such as stainless steel mesh. The modified stainless steel mesh also exhibits the same excellent water roll-off property. (A demonstration video can be found in the ESI. $\dagger$ ) The hydrophobicity of the coating and water repellency persisted for an extended length of time, enabling the various modified mesh, including PP mesh and stainless steel mesh, to spontaneously float on the water-air interface. The floating mesh prevents itself from sinking into the water.

The floating mesh ensures that the pores of the PPy-coated mesh are unblocked to allow continuous optimized flow flux of water vapor (Fig. 3A). The photothermal property of the PPycoated PP mesh was evaluated by in situ temperature mapping using an IR camera under simulated sunlight with a light intensity of $1000 \mathrm{~W} \mathrm{~m}^{-2}$ (Fig. 3B). Under solar irradiation, the temperature of PPy-coated PP mesh gradually increased, reaching an equilibrium temperature of $\sim 50{ }^{\circ} \mathrm{C}$ after $\sim 600 \mathrm{~s}$. In darkness, recovery back to $22{ }^{\circ} \mathrm{C}$, roughly room temperature, is achieved in another $\sim 600 \mathrm{~s}$. In comparison, the PP mesh without PPy coating remains at $20^{\circ} \mathrm{C}$ under 20 minutes of solar irradiation without increase in temperature. The in situ temperature increase clearly demonstrated the efficient photothermal conversion property of PPy-coated PP mesh due to the contribution of the $\sim 300 \mathrm{~nm}$ PPy coating on the PP mesh. Due to the low thermal conductivity and low specific heat capacity of
PPy and PP material, the PPy-coated mesh forms a heat barrier, enhancing heat localization. ${ }^{27}$

Enhanced water evaporation was then evaluated by floating hydrophobic PPy-coated PP mesh at the water-air interface inside the beaker. Sunlight irradiated the water at an intensity of $1000 \mathrm{~W} \mathrm{~m}^{-2}$. The mass loss of water due to evaporation was monitored by an analytical balance. As a control, the mass loss of water with the floating unmodified PP mesh at the air-water interface and the mass loss of water in the absence of mesh on the water surface were also measured. Seawater evaporation rate follows the same trend. Using PPy-coated PP mesh enhances the rate of the seawater evaporation. Solar vapor generation efficiency is defined as the ratio of enthalpy change in the generated vapor divided by total incoming solar flux, which is evaluated based on the water evaporation rate,

$$
\begin{array}{r}
\eta=Q(\text { water evaporation }) / Q_{\text {light }}= \\
\quad\left(\text { water evaporation rate } \times H_{\mathrm{e}}\right) / Q_{\text {light }}
\end{array}
$$

where $Q_{\text {light }}$ is the incident light intensity $\left(1000 \mathrm{~W} \mathrm{~m}^{-2}\right)$ and $H_{\mathrm{e}}$ is the heat of evaporation of water $\left(2260 \mathrm{~kJ} \mathrm{~kg}^{-1}\right){ }^{9}$ As calculated, the solar vapor generation efficiency of the PPy-coated PP mesh is higher than that when using PP mesh or pure solution for both water and seawater evaporation. The PPy-coated PP mesh has a solar vapor generation efficiency of $72 \%$, which is slightly higher than that achieved with stainless steel mesh with PPy coatings in micrometer thickness ${ }^{9}$ and much higher than that of commercialized solar stills with typical efficiencies from $24 \%$ 


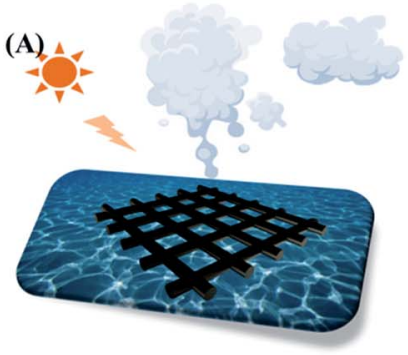

(D)

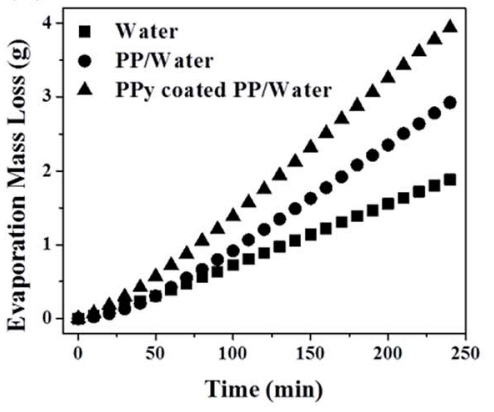

(B)

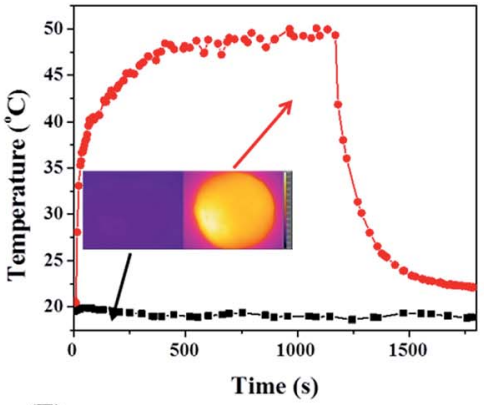

(E)

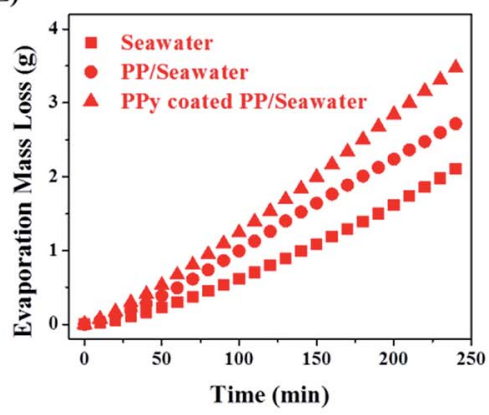

(C) Hydrophobic PPy coated PP mesh

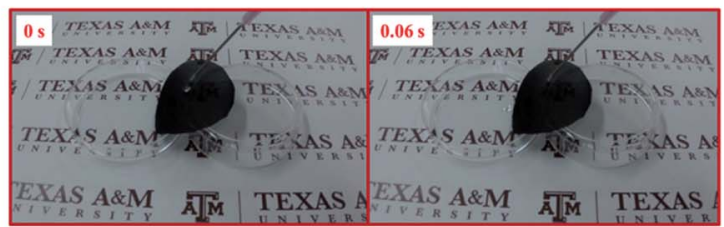

(F)

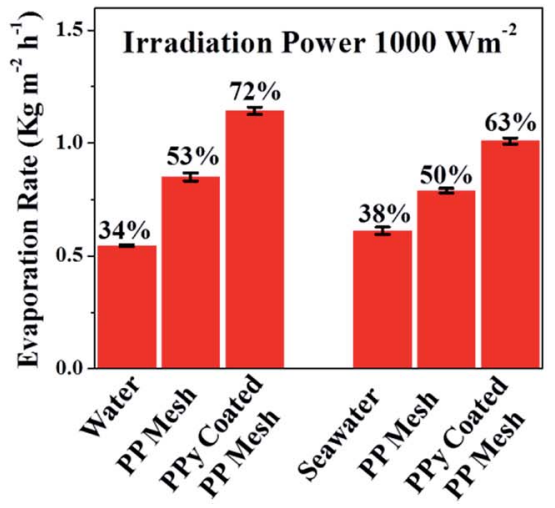

Fig. 3 Interfacial solar membrane for water evaporation. (A) Illustration of interfacial solar membrane for water evaporation. Solar irradiation increases localized temperature on the mesh, causing the water vapor to form clouds of condensed fresh water. (B) Photothermal heating and cooling of PP and PPy-coated PP mesh under 30 min irradiation. Inset image is the temperature distribution of PP and PPy-coated PP mesh after 2 min irradiation. The radiation was turned off and the temperature was allowed to gradually decrease. (C) Excellent water repellency of water droplets on the hydrophobic PPy-coated PP mesh. (Video in the ESI.†) The water droplet rolls off the surface within $0.1 \mathrm{~s}$. (D) Solar evaporation of pure water and (E) solar evaporation of seawater $(35000$ ppm NaCl) without mesh as well as with floating PP mesh and floating PPy-coated PP mesh. (F) Evaporation rate and solar vapor generation efficiency under different conditions (left to right: pure water, floating PP mesh, and floating PPy-coated PP mesh on the water, pure seawater (without mesh), floating PP mesh, and floating PPy-coated PP mesh on the seawater). The light intensity was fixed at $1000 \mathrm{~W} \mathrm{~m}^{-2}$. The mesh area is $861 \mathrm{~mm}^{2}$.

to $45 \% .{ }^{17}$ The higher efficiency achieved in our experimental mesh is because the efficiency in harvesting solar energy is conferred by the $\sim 300 \mathrm{~nm}$ PPy coating as well as the heat barrier property of the bulk PPy-coated PP mesh. The heat barrier is attributable to low thermal conductivity and low specific heat capacity of both PPy coating and PP mesh. Moreover, the PPy thin film coating is robust under mild stirring in water and seawater $(35000 \mathrm{ppm} \mathrm{NaCl})$. It is stable when floating or immersed in water and seawater under mild stirring for 7 days. The FTIR spectra of the selected solutions do not show any characteristic peaks from PPy or possible decomposed structure (see ESI $\uparrow$ ), which indicates that neither distinguishable detachment and release from the PPy coating nor the decomposition of the PPy coating occurred.

\section{Conclusions}

In conclusion, we have developed a new kind of interfacial solar thermal membrane based on PPy thin film coating on PP mesh with potential application as a thermal desalination membrane that uses solar energy as a heat source to vaporize seawater and brackish water. First, PPy was selected due to its broad spectrum absorption, which is closely matched to the solar radiation spectrum, high photo-to-thermal conversion efficiency, and outstanding stability. PPy is inexpensive compared to noble metal nanoparticles and exhibits good biocompatibility and low long-term cytotoxicity as compared to carbon materials. Second, the PPy thin film fabrication technology requires a low energy input, is environmentally friendly, allows a PPy thin film to tightly coat regardless of the curvature and conductivity, and a variety of substrates, such as non-conductive flexible PP mesh, which has been previously used in thermal desalination. After coating $\sim 300 \mathrm{~nm}$ PPy thin film on PP mesh, the solar vapor generation efficiency was measured to be $72 \%$, which was much higher than that of the commercialized solar stills, with typical efficiencies from $24 \%$ to $45 \%$. Moreover, the polypyrrole thin film retains good adhesion under mild flow of water and seawater. This membrane opens doors to the next generation of thermal desalination membrane utilizing solar energy.

\section{Acknowledgements}

The authors would like to acknowledge Dr Manuel Marquez and YNano LLC for their support of X. H.'s colloidal soft-assembly research. This work is also partially supported by NASA (NASA-NNX13AQ60G). The authors acknowledge the Microscopy and Imaging Center (MIC) at Texas A\&M University for access to the FE-SEM and TEM. The FE-SEM instrument was supported by the National Science Foundation under Grant DBI-0116835. The authors also thank Dr Micah J. Green and Brandon Sweeney for assistance with IR camera measurement and Dr Mustsfa Akbulut for use of the contact angle goniometer. 


\section{References}

1 M. Elimelech and W. A. Phillip, Science, 2011, 333, 712-717. 2 M. A. Shannon, P. W. Bohn, M. Elimelech, J. G. Georgiadis, B. J. Marinas and A. M. Mayes, Nature, 2008, 452, 301-310.

3 R. Yang, H. Jang, R. Stocker and K. K. Gleason, Adv. Mater., 2014, 26, 1711-1718.

4 R. Semiat, Environ. Sci. Technol., 2008, 42, 8193-8201.

5 O. Neumann, A. S. Urban, J. Day, S. Lal, P. Nordlander and N. J. Halas, ACS Nano, 2013, 7, 42-49.

6 O. Neumann, C. Feronti, A. D. Neumann, A. Dong, K. Schell, B. Lu, E. Kim, M. Quinn, S. Thompson, N. Grady, P. Nordlander, M. Oden and N. J. Halas, Proc. Natl. Acad. Sci. U. S. A., 2013, 110, 11677-11681.

7 O. Neumann, A. D. Neumann, E. Silva, C. Ayala-Orozco, S. Tian, P. Nordlander and N. J. Halas, Nano Lett., 2015, 15, 7880-7885.

8 Y. Liu, S. Yu, R. Feng, A. Bernard, Y. Liu, Y. Zhang, H. Duan, W. Shang, P. Tao, C. Song and T. Deng, Adv. Mater., 2015, 27, 2768-2774.

9 L. Zhang, B. Tang, J. Wu, R. Li and P. Wang, Adv. Mater., 2015, 27, 4889-4894.

10 G. Ni, G. Li, S. V. Boriskina, H. Li, W. Yang, T. Zhang and G. Chen, Nat. Energy, 2016, 1, 16126.

11 W. Shang and T. Deng, Nat. Energy, 2016, 1, 16133.

$12 \mathrm{M}$. Khayet and T. Matsuura, in Membrane Distillation, Elsevier, Amsterdam, 2011, pp. 17-40.
13 Z. Zha, X. Yue, Q. Ren and Z. Dai, Adv. Mater., 2013, 25, 777782.

14 E. Y. Hleb and D. O. Lapotko, Nanotechnology, 2008, 19, 355702.

15 A. Nel, T. Xia, L. Mädler and N. Li, Science, 2006, 311, 622627.

16 M. Gryta, Membranes, 2012, 2, 415-429.

17 A. E. Kabeel and S. A. El-Agouz, Desalination, 2011, 276, 1-12. 18 Y. Tan and K. Ghandi, Synth. Met., 2013, 175, 183-191.

19 P. Kim, T.-S. Wong, J. Alvarenga, M. J. Kreder, W. E. AdornoMartinez and J. Aizenberg, ACS Nano, 2012, 6, 6569-6577.

20 D. F. Acevedo, E. Frontera, M. F. Broglia, F. Mücklich, M. C. Miras and C. A. Barbero, Adv. Eng. Mater., 2011, 13, 405-410.

21 M. M. Ayad, Adv. Eng. Mater., 1994, 32, 9-14.

22 X. Huang, J. D. Chrisman and N. S. Zacharia, ACS Macro Lett., 2013, 2, 826-829.

23 X. Huang and N. S. Zacharia, J. Appl. Polym. Sci., 2015, 132, 42767.

24 X. Huang and N. S. Zacharia, Soft Matter, 2013, 9, 7735-7742.

25 D. Mecerreyes, V. Alvaro, I. Cantero, M. Bengoetxea, P. A. Calvo, H. Grande, J. Rodriguez and J. A. Pomposo, Adv. Mater., 2002, 14, 749-752.

26 S. Akerboom, S. P. Pujari, A. Turak and M. Kamperman, ACS Appl. Mater. Interfaces, 2015, 7, 16507-16517.

27 Y. Ito, Y. Tanabe, J. Han, T. Fujita, K. Tanigaki and M. Chen, Adv. Mater., 2015, 27, 4302-4307. 